\title{
Microscopic Examination of Findings Encountered during Cadaver Dissection: Malignant, Benign or Anatomic Variation?
}

\author{
Maria Cicioni \\ Thomas Jefferson University \\ Vandi Ly \\ Thomas Jefferson University \\ Guiyun Zhang \\ Thomas Jefferson University \\ Bruce Fenderson \\ Thomas Jefferson University
}

Follow this and additional works at: https://jdc.jefferson.edu/pacbfp

Part of the Medical Anatomy Commons, and the Pathology Commons

Let us know how access to this document benefits you

\section{Recommended Citation}

Cicioni, Maria; Ly, Vandi; Zhang, Guiyun; and Fenderson, Bruce, "Microscopic Examination of Findings Encountered during Cadaver Dissection: Malignant, Benign or Anatomic Variation?" (2017). Department of Pathology, Anatomy, and Cell Biology Faculty Papers. Paper 223. https://jdc.jefferson.edu/pacbfp/223

This Article is brought to you for free and open access by the Jefferson Digital Commons. The Jefferson Digital Commons is a service of Thomas Jefferson University's Center for Teaching and Learning (CTL). The Commons is a showcase for Jefferson books and journals, peer-reviewed scholarly publications, unique historical collections from the University archives, and teaching tools. The Jefferson Digital Commons allows researchers and interested readers anywhere in the world to learn about and keep up to date with Jefferson scholarship. This article has been accepted for inclusion in Department of Pathology, Anatomy, and Cell Biology Faculty Papers by an authorized administrator of the Jefferson Digital Commons. For more information, please contact: JeffersonDigitalCommons@jefferson.edu. 


\section{Microscopic Examination of Findings Encountered during Cadaver Dissection: Malignant, Benign or Anatomic Variation?}

\author{
Cicioni M, Ly V, Zhang G and Fenderson BA* \\ Department of Pathology, Anatomy and Cell Biology, \\ Thomas J efferson University, USA \\ *Corresponding author: Bruce A. Fenderson, \\ Department of Pathology, Anatomy and Cell Biology, \\ Thomas J efferson University, 1020 Locust Street, Room \\ 263 J efferson Alumni Hall, Philadelphia, PA 19107. USA
}

Received: January 19, 2017; Accepted: March 20, 2017; Published: March 29, 2017

\begin{abstract}
Pathologic findings encountered during cadaver dissection provide an opportunity for integrating the preclinical basic sciences and encouraging critical thinking. The objective of this study was to determine whether it is possible to make a pathologic diagnosis of an unknown mass from an embalmed cadaver. Diagnoses would have to be based solely on gross and microscopic appearance of tissue, without clinical histories of the cadaveric donors. The tissue samples we removed from each mass were surprisingly well preserved and showed minimal autolysis. Indeed, some of the histological detail was as clear as may be found in any textbook. We were able to obtain a pathologic diagnosis for 6 cases that illustrate complications of malignant neoplasms arising in the colon, breast, ovary, and kidney. Our results emphasize the importance of integrating gross and microscopic anatomy with pathology to facilitate a comprehensive understanding of disease. This histopathology independent learning project could become an integral part of dissection-based anatomy courses, and stimulate students to become more inquisitive when they see something out of the ordinary in their cadaver.
\end{abstract}

Keywords: Medical education; Cadaver dissection; Gross anatomy; Pathology; Ovarian cancer; Breast cancer; Metastatic cancer

\section{Abbreviations}

H\&E: Hematoxylin and Eosin

\section{Introduction}

Pathologic findings, anatomic variations, and evidence of past surgical procedures are frequently encountered during cadaver dissection [1-4]. These unexpected findings provide a valuable opportunity for active learning that integrates the preclinical basic sciences and stimulates the development of critical thinking skills [5]. To promote independent learning, students at Thomas Jefferson University are encouraged to investigate unexpected findings in their cadavers during dissection. However, upon gross inspection, it may remain unclear whether these discoveries are pathologic or the result of variability in anatomic development.

Previously, we described two cases that provided excellent opportunities for integrating anatomy with pathology and clinical medicine [4]. The first case was a massive ovarian tumor that displaced the urinary bladder and uterus, and compressed the ureters and rectum. The second case appeared to be breast cancer with widespread metastases. Students discussed clinical complications of these cases and reviewed clinicopathologic findings that differentiate benign from malignant neoplasms. Despite the interest that these cases generated, in terms of class discussion and independent study, gross inspection of the tumors did not permit us to establish a pathologic diagnosis.

The objective of this study, a medical student honors project, was to determine whether it is possible to make a pathologic diagnosis of an unknown mass from a cadaver, in which the preservation of organs and tissues following routine embalming, dissection, and storage was unknown. Without good preservation of cellular and nuclear morphology, it is difficult (if not impossible) to determine the pathologic basis of disease. Moreover, our diagnoses would have to be based solely on gross and microscopic appearance of the tissues, without access to clinical histories of the cadaveric donors, including age, past medical history, and proposed cause of death.

\section{Methods}

Anatomy instruction at Sidney Kimmel Medical College of Thomas Jefferson University is based on overview lectures and cadaver dissection [6]. Cadavers were obtained from the Humanity Gifts Registry, embalmed using "Maryland State Anatomical Solution" (Hydrol Chemical Company), and maintained at $10^{\circ} \mathrm{C}$. During dissection, students documented anatomic variations and pathologic findings. Organs with evidence of gross pathology were removed at the end of the course and kept moist with water containing $10 \%(\mathrm{v} / \mathrm{v})$ propylene glycol. A total of 6 cases were selected for this Pathology Honors project: ovary $(\mathrm{n}=1)$, kidney $(\mathrm{n}=1)$, liver $(\mathrm{n}=1)$, and lung $(n=3)$. Paraffin embedded tissues were sectioned at 6 microns and stained with Hematoxylin and Eosin (H\&E). Slides were examined using a Nikon light microscope at 2x, 10x, and 20x magnification, and photographed using a digital camera.

\section{Results}

\section{Case 1: Lung with multiple, white pleural plaques}

Gross examination of the hypoplastic, right lung from a cadaver 


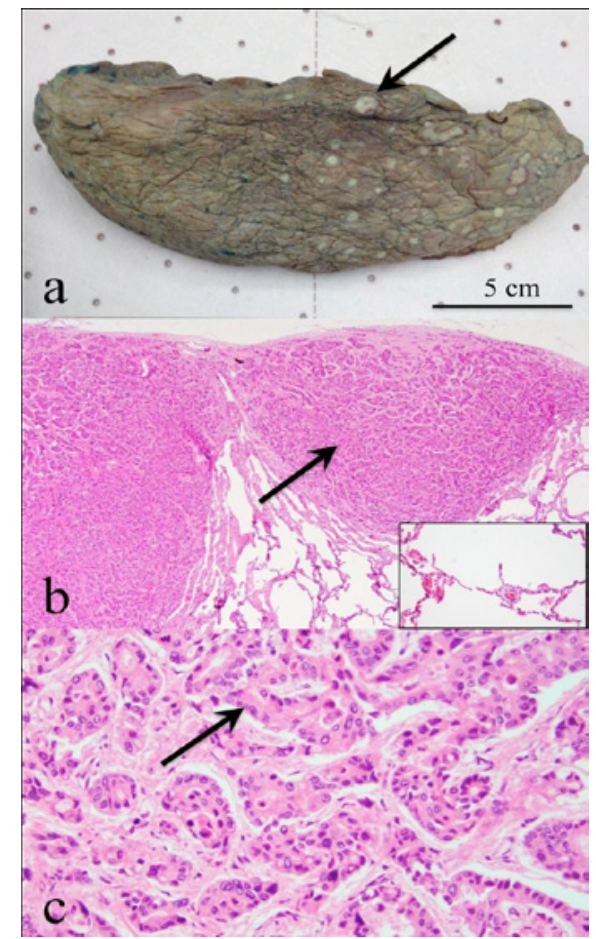

Figure 1: Gross and microscopic examination of a right lung from a cadaver with widespread abdominal metastases. The hypoplastic lung exhibits multiple, white plaques on the visceral pleura (1a, arrow). Microscopic examination of a pleural plaque reveals small, well-circumscribed pleural nodules (1b at $\mathbf{2 x}$, arrow) surrounded by normal pulmonary tissue. The inset shows well-preserved pulmonary alveoli. At higher magnification, the tumor nodules show well-differentiated glands (1c at 20x, arrow) and extensive interstitial fibrosis. Some of the glandular lumens contain characteristic necrotic debris, referred to as "dirty necrosis". These gross and microscopic findings are consistent with a diagnosis of metastatic adenocarcinoma from the colon.

with widespread abdominal metastases reveals multiple, white, palpable plaques on the visceral pleura (Figure 1a). Biopsy and microscopic examination of one pleural plaque demonstrates wellcircumscribed pleural nodules surrounded by normal pulmonary tissue (Figure $1 \mathrm{~b}$ ). On average, the tumor nodules measured $0.5 \mathrm{~cm}$. The presence of detailed alveolar histology in this cadaveric specimen (Figure $1 \mathrm{~b}$, inset) indicates that the embalming process was sufficient to preserve tissues with morphology suitable for histopathologic analysis. At higher magnification, the tumor nodules show welldifferentiated glands (Figure 1c) and extensive interstitial fibrosis. These findings suggest a "low-grade carcinoma with desmoplasia". Some of the glandular lumens contain characteristic necrotic debris, referred to as "dirty necrosis". Together, these gross and microscopic findings are consistent with a diagnosis of metastatic adenocarcinoma from the colon. Although immunohistochemical assays were not performed as part of this study, CK20 is an intracellular tumor marker that could be used to distinguish primary from secondary (metastatic) adenocarcinoma [7].

\section{Case 2: Massive ovarian tumor}

As reported previously [4], dissection of an elderly female cadaver revealed a large tumor mass filling the entire true pelvis. The isolated tumor exhibits a smooth connective tissue capsule and measures

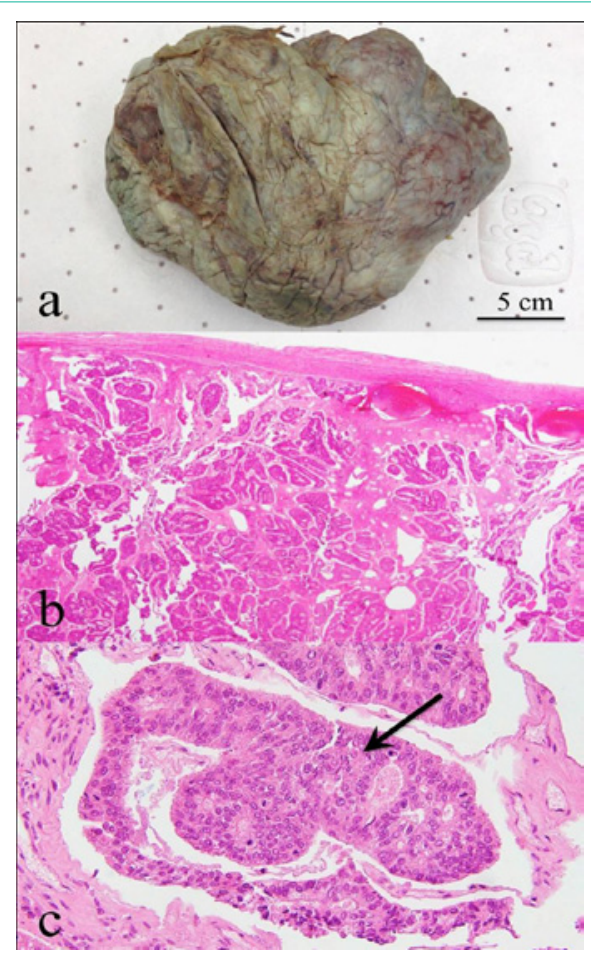

Figure 2: Gross and microscopic examination of a massive ovarian tumor The isolated tumor exhibits a smooth connective tissue capsule and measures $22 \times 15 \times 15 \mathrm{~cm}(2 a)$. Incision reveals small cystic spaces that suggest a benign cystadenoma [4]; however, microscopic examination reveals welldifferentiated glands resembling uterine endometrium $(\mathbf{2 b}$ at $\mathbf{2 x}$ and $2 \mathrm{c}$ at 20x, arrow) consistent with a diagnosis of endometrioid adenocarcinoma of the ovary.

$22 \times 15 \times 15 \mathrm{~cm}$ (Figure 2a). Incision reveals small cystic spaces that suggest a benign ovarian cystadenoma. However, microscopic examination reveals well-differentiated glands resembling uterine endometrium (Figure $2 \mathrm{~b} \& 2 \mathrm{c}$ ). This finding is consistent with a diagnosis of endometrioid adenocarcinoma of the ovary. The current belief is that these neoplasms are derived from foci of endometriosis that undergo malignant transformation [8]. During the reproductive years, the endometrial epithelium is subjected to repeated cycles of injury and repair that increase the risk for mutations that predispose to endometriosis and/or neoplasia.

\section{Case 3: Solitary renal mass}

Gross examination of a cadaver kidney reveals an encapsulated exophytic mass measuring $1.5 \times 1.5 \mathrm{~cm}$ (Figure 3a). Incision through the capsule exposed a highly vascular and hemorrhagic tumor. Microscopic examination reveals malignant cells with clear cytoplasm (Figure $3 \mathrm{~b}$ ) surrounded by normal renal glomerular and tubular tissue (Figure 3c). Together, these findings are consistent with a diagnosis of clear cell variant of renal cell carcinoma. The vascularity of the tumor noted on gross inspection supports this diagnosis, given that renal cell carcinomas typically metastasize via a hematogenous route. Most cases of renal cell carcinoma are associated with loss of heterozygosity and/or driver mutations in the VHL protooncogene [8].

\section{Case 4: Liver with cannonball metastases}

Gross examination of an enlarged liver reveals multiple "cannonball metastases" from a tumor of unknown origin. The 


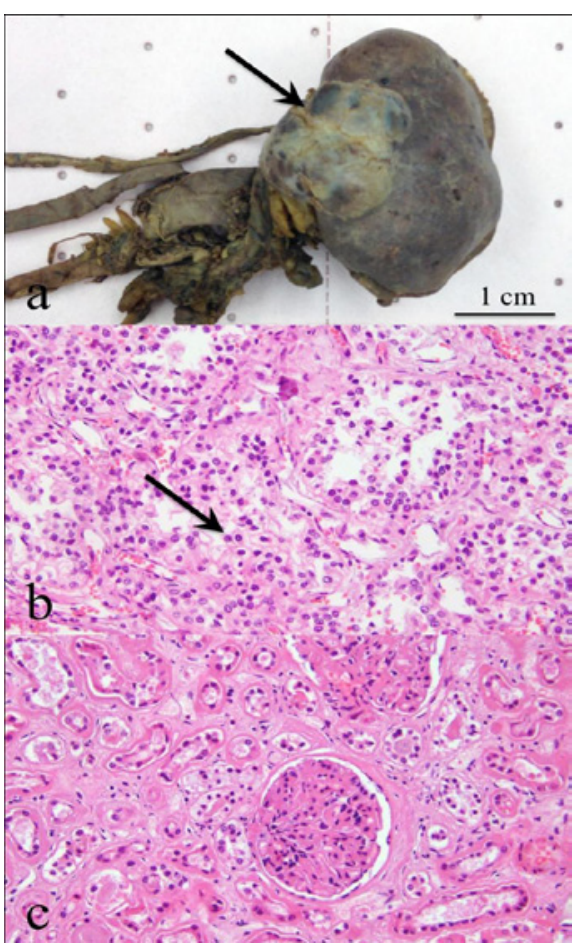

Figure 3: Gross and microscopic examination of a solitary renal mass. Gross findings include an encapsulated exophytic mass on the kidney surface measuring $1.5 \times 1.5 \mathrm{~cm}$ (a, arrow). Incision through the capsule exposes a highly vascular and hemorrhagic tumor. Microscopic examination reveals malignant cells with clear cytoplasm (3b at 20x, arrow) surrounded by norma renal glomerular and tubular tissue (3c at 20x). These findings are consistent with a diagnosis of clear cell variant of renal cell carcinoma.

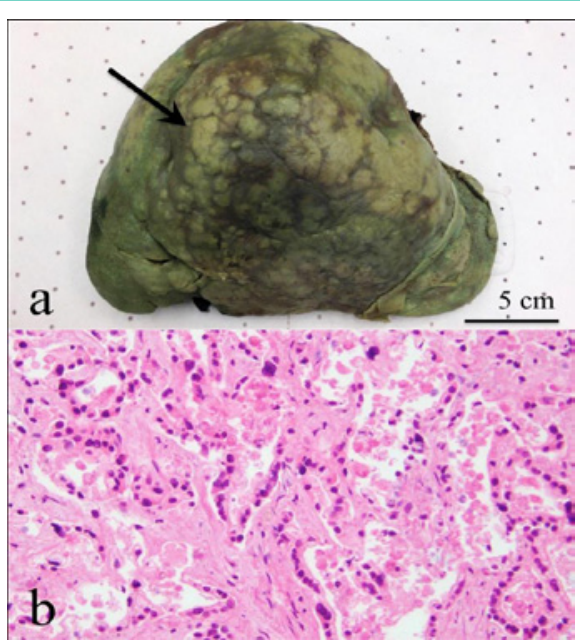

Figure 4: Gross and microscopic examination of an enlarged liver with "cannonball metastases". The cadaver liver appears dark green (stained with bile) and shows numerous metastatic tumor nodules present on the surface and scattered throughout the hepatic parenchyma (4a, arrow). Microscopic examination reveals low-grade adenocarcinoma resembling colonic glands. Considerable autolysis is observed, along with necrotic cellular debris (4b at 20x). Together, these gross and microscopic findings are consistent with a diagnosis of metastatic adenocarcinoma from the colon.

cadaveric liver appears dark green (stained with bile). Metastatic tumor nodules are present on the external surface and scattered throughout the hepatic parenchyma (Figure 4a). On average, the

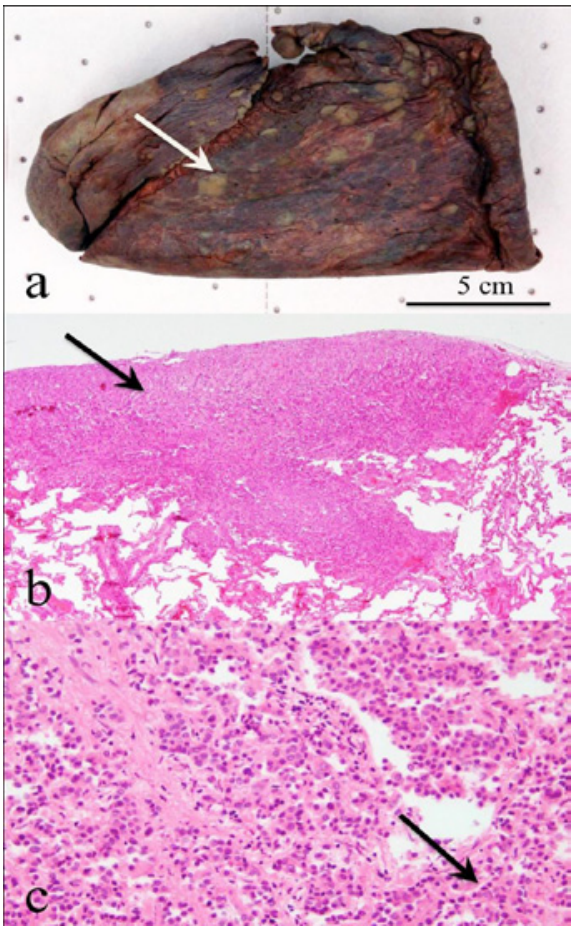

Figure 5: Gross and microscopic examination of the right lung from a cadaver with evidence of widespread metastases and a unilateral mastectomy. Gross findings include multiple, tan plaques on the visceral pleura (5a). Microscopic examination reveals small, well-circumscribed pleural nodules $\mathbf{( 5 b}$ at $\mathbf{2} \mathbf{x}$, arrow). At higher magnification, the nodules show invasive carcinoma of the breast with characteristic "single file" growth pattern (5c at 20x, arrow). This growth pattern is consistent with a diagnosis of metastatic invasive lobular carcinoma of the breast.

tumor nodules measured $1 \mathrm{~cm}$. Microscopic examination reveals low-grade adenocarcinoma resembling colonic glands. Considerable autolysis is observed, along with necrotic cellular debris (Figure 4b). Together, these gross and microscopic findings are consistent with a diagnosis of metastatic adenocarcinoma from the colon. The liver is a common site of metastasis of malignant tumors of the gastrointestinal tract [8].

\section{Case 5: Lung with multiple, tan pleural plaques}

As reported previously [4], dissection of the pectoral region of an elderly female cadaver with signs of cachexia revealed a unilateral mastectomy with silicone breast implant. Further exploration of the thorax and abdomen revealed widespread tumor metastases. Gross examination of the right lung reveals multiple, tan plaques on the visceral pleura (Figure 5a). Microscopic examination reveals small, well-circumscribed pleural nodules (Figure 5b). At higher magnification, the nodules show invasive carcinoma of the breast with a characteristic "single file" growth pattern (Figure $5 \mathrm{c}$ ). This distinctive growth pattern is consistent with a diagnosis of metastatic invasive lobular carcinoma of the breast. Driver mutations in the E-cadherin gene (CDH-1) are associated with this type of breast cancer [9]. Approximately 50\% of female $\mathrm{CDH} 1$ mutation carriers are expected to develop invasive lobular carcinoma of breast owing to lack of this cell-cell adhesion molecule. A diagnosis of metastatic breast cancer was also made in a separate lung biopsy (cadaver case 6, data not shown). 


\section{Discussion}

Our results demonstrate that it is possible to make a pathologic diagnosis of an unknown mass obtained from a cadaver in the anatomy dissection laboratory. Embalming and storage of human gifts do not provide an obstacle to histopathology. Similar findings have been reported previously $[10,11]$. The tissue samples we removed from each mass were surprisingly well preserved. For example, histology of the normal alveolar-capillary interface (Figure 1) and kidney parenchyma (Figure 3) was excellent - as detailed as may be seen in histology textbooks. We were able to make a pathologic diagnosis from all 6 tumors that we biopsied. The cases described here illustrate complications of malignant neoplasms arising in the colon, breast, ovary, and kidney. Four of these cases involved metastatic cancer. Our findings emphasize the crucial importance of correlating gross and microscopic anatomy for understanding the pathologic basis of disease.

This study represents an independent learning project for a medical student (Mariah Cicioni) participating in the Pathology Program for Advanced Study. The Pathology Program for Advanced Study at Thomas Jefferson University is an enrichment experience for second-year medical students with ability and interest. Approximately 25 students per year qualify for the Honors Program by scoring in the top $10 \%$ of their class on the Foundations of Pathology final course examination. Each student has a faculty mentor. Students are encouraged to attend various pathology conferences and shadow their faculty mentor. Students develop and present a clinical case report or basic science/translational research findings at a year-end "Pathology Honors Student Research Symposium". Characteristics of medical students completing the Jefferson Pathology Honors Program were described previously [12].

This self-directed research project will, hopefully, stimulate future students to become more inquisitive when they see something out of the ordinary in their cadaver. In reflection, Mariah writes: "This project helped encourage me to always remain curious and keep asking questions. Jefferson's faculty and staff spend four years teaching us the answers and how to find them. But after beginning the clinical years, it has become evident to me that sometimes the greater challenge is starting out with a good question and having the confidence to ask it. By giving medical students the opportunity to design an independent project, schools can help instill this invaluable skill in future physicians".

In summary, pathologic findings and anatomic variations encountered during cadaver dissection provide an opportunity for students to pursue scholarly inquiry. We believe that independent study projects, such as this one, could become incorporated into gross anatomy courses in medical school. Now that we know tissue samples can be stained with H\&E and examined by light microscopy, it would be interesting to see if similar projects could be taken a step further by adding immunohistochemical assays and genetic testing. All medical students at Sidney Kimmel Medical College of Thomas Jefferson University are now expected to pursue one or more scholarly projects during their undergraduate medical training. The goal is to provide students with an opportunity to: i) learn how to critically evaluate new research findings for the benefit of patient care; and ii) participate in research leading to the creation of new knowledge. In addition, it is believed that an emphasis on scholarship in the medical curriculum will help nurture holistic competencies expected of an outstanding physician, including professionalism and commitment to life-long learning.

\section{Acknowledgment}

The authors acknowledge the support of Dr. Stephen Peiper, Chair of Department of Pathology, Anatomy and Cell Biology at Thomas Jefferson University. We thank Christopher Marcucci for managing the Anatomy Dissection Facility and Dr. Constantine Daskalakis, Director of Undergraduate Medical Scholarship at Thomas Jefferson University, for helpful comments on the manuscript. We gratefully acknowledge the Intersociety Council for Pathology Information (ICPI) for their financial support of the Pathology Honors Program in the Department of Pathology, Anatomy and Cell Biology at Thomas Jefferson University. Most importantly, we wish thank the Humanity Gifts Registry and the many families who donate bodies of their loved ones for training the next generation of medical caregivers. Humanity Gifts Registry is a non-profit agency of the Commonwealth of Pennsylvania concerned primarily with the receipt and distribution of bodies donated to all medical and dental schools in the state for teaching purposes.

\section{References}

1. Wood A, Struthers K, Whiten S, Jackson D, Herrington CS. Introducing gross pathology to undergraduate medical students in the dissecting room. Anat Sci Educ. 2010; 3: 97-100.

2. Alyafi MM, Al Amodi AA, Juurlink BHJ, Ganguly P. How the dissection laboratory facilitates integration of learning: presence of abdominal aortic aneurysm with a large intracardiac thrombus: a rare cadaver finding. Int $\mathrm{J}$ Angiol. 2012; 21: 77-80.

3. Zhang G, Fenderson BA. Bilateral accessory flexor muscle of the forearm giving rise to a variant head of the first lumbrical. Int J Anat Var. 2014; 12.

4. Zhang G, Fenderson BA. Pathology encountered during cadaver dissection provides an opportunity for integrated learning and critical thinking. Austin $\mathrm{J}$ Anat. 2014; 1 : 3.

5. Bartels D. What is Your Question? Scientific American. 2013.

6. Zhang G, Fenderson BA, Schmidt R, Veloski JJ. Equivalence of students scores on timed and untimed anatomy practical examinations. Anat Sci Educ. 2013; 6: 281-285.

7. Gurda GT, Zhang L, Wang Y, Chen L, Geddes S, Cho WC, et al. Utility of five commonly used immunohistochemical markers TTF-1, Napsin A, CK7, CK5/6 and P63 in primary and metastatic adenocarcinoma and squamous cell carcinoma of the lung: a retrospective study of 246 fine needle aspiration cases. Clin Trans Med. 2015; 4: 16.

8. Rubin's Pathology: Clinicopathologic Foundations of Medicine 7th edition (D Strayer and E Rubin, editors), Wolters Kluwer, Philadelphia, 2014.

9. Dossus L, Benusiglio PR. Lobular breast cancer: incidence and genetic and non-genetic risk factors. Breast Cancer R. 2015; 17: 37

10. Baker MG, Bradley EB, McCollum MA, Rusell MA. The cadaveric skin biopsy project: description and student evaluation of an innovative approach to dermatology instruction in the preclerkship medical school curriculum. J Am Acad Dermatol. 2014; 71; 314-319.

11. Wood A, Whiten S, McVee J, Issberner J, Jackson D, Herrington CS. Histopathology from the dissecting room: Are cadavers a suitable source of educationally useful histopathology specimens? Anatomy. 2015; 9.

12. Fenderson BA, Hojat M, Damjanov I, Rubin E. Characteristics of medical students completing an honors program in pathology. Human Path. 1999; 30: 1296-1301. 